\title{
Membrane Stress Proclivities in the Mammalian Labyrinth
}

\author{
Daniel J. Pender ${ }^{1}$ \\ ${ }^{1}$ Department of Otolaryngology, Columbia University, New York City, \\ New York, United States \\ Int Arch Otorhinolaryngol 2014;18:398-402.
}

\begin{abstract}
Address for correspondence Daniel J. Pender, MSE, MD, Department of Otolaryngology, Columbia University, 145 West 86th Street Suite 1C, New York City, NY 10024, United States (e-mail: djp2@columbia.edu).
\end{abstract}

\begin{abstract}
Introduction The membranes of the inferior division of the labyrinth in some mammals appear more vulnerable to hydropic distention than those of the superior division. This finding in guinea pigs, cats, and humans has been attributed to the evidently thinner membranes with implied higher stress levels.

Objective The objective of this study is to identify other configurational features, if any, that may contribute to membrane stress proclivity and therefore might act to augment or ameliorate stress in the several chambers of the membranous labyrinth. Methods Stress proclivity can be investigated using shell theory to analyze a geometric model of the labyrinthine membranes in mammals. Such an approach can provide the necessary mathematical descriptions of stress in each chamber of the labyrinth.

Results Stress proclivity depends on three physical features: membrane thickness, radial size, and chamber shape. Lower stress proclivities are projected for smaller chambers with thick, highly synclastic membranes. Higher stress levels are projected for larger chambers with thin, flat, or anticlastic membranes.

Keywords

- stress

- hydrops

- labyrinth

- Meniere disease

Conclusions In the mammalian labyrinth, pars superior chambers exhibit permutations of membrane thickness, size, and favorable shapes that reduce stress proclivity. In contrast, the pars inferior chambers are characterized by thin membranes with flat contours and adverse shapes that make them especially vulnerable to hydropic distention.
\end{abstract}

\section{Introduction}

The mammalian labyrinth exhibits chambers with a variety of shapes and sizes, cobbled together evolutively into a complex asymmetric structure. ${ }^{1}$ Human temporal bone studies in cases of Meniere disease have revealed that the inferior division of the membranous labyrinth is much more vulnerable to hydropic distention than the superior division. ${ }^{2}$ This is also the case in experimentally induced hydrops in other mammals, such as guinea pigs and cats. ${ }^{3}$ This has previously been ascribed to raised endolymphatic pressure acting to dilate the visibly thinner membranes of the inferior labyrinth. ${ }^{4}$ The details of this mechanism remain to be clarified.
A mechanics of materials approach can be used to investigate stress and strain in these membranes. Stress assessment focuses on how a load is distributed in a structure, and strain assessment focuses on how the structure deforms in response to the loading. This study will concern itself with stress proclivity in the labyrinth (i.e., the tendency for membrane stress to mount as transmural pressure increases). Strain and membrane compliance considerations are beyond the scope of the current study and will be addressed separately in a subsequent report. Variation in stress proclivity among the several chambers due to the differences in configuration may play a role in the observed hydropic findings. received

May 28, 2014

accepted

June 21, 2014

published online

August 25, 2014
DOI http://dx.doi.org/

10.1055/s-0034-1385846. ISSN 1809-9777.
Copyright $(\underset{0}{ } 2014$ by Thieme Publicações License terms Ltda, Rio de Janeiro, Brazil

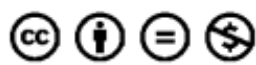




\section{Methods}

Stress proclivity, defined as stress per unit pressure in the baseline nondistended state, can be investigated analytically through the use of a geometric model of the labyrinthine membranes in mammals. ${ }^{5}$ The complete model employs the following geometries: tori for the semicircular canals, spheroids for the ampullae, cylinders for the utricle and crus commune, discus for the saccule, and spiral torus for Reissner membrane in the cochlea duct. Stress proclivities in the various geometric chambers can be estimated through a micromechanical static force analysis. Such analysis is based on membrane theory to relate internal pressure to wall tension in the labyrinth. The general relationship is given in Eq. 1.

$$
p=T_{a} \cdot C_{a}+T_{h} \cdot C_{h}
$$

where $p$ is transmural pressure on the membrane; $T_{a}$ is axial tension in the membrane; $T_{h}$ is hoop tension in the membrane; $C_{a}$ is axial curvature of the membrane; $C_{h}$ is hoop curvature of the membrane.

In general, applicability of this relationship assumes that the membranes are thin with a 10:1 ratio between chamber diameter and wall thickness and that the membranes offer no bending resistance. ${ }^{6}$ Details of the derivations of the equations of stress proclivity for the several chamber geometries are presented elsewhere. ${ }^{7}$ These equations are presented below. The abbreviations used in these equations are summarized in -Table 1.

Applied to the discoid (extreme oblate spheroid) shape used to emulate the saccule, membrane theory indicates that maximal stress is meridional and occurs at the polar region. Its value is given by Eq. 2 :

$$
t_{\text {meridional } \max }=\{r / 2 l\}(r)(1 / w)(p)
$$

Note that when axial length $l$ is very small in relation to $r$, the oblate spheroid is very flat and discoid, and the value of the curly bracket term is very large, implying very high stress for the discoid shape. In contrast, when the $r$ and $l$ are equal, the oblate spheroid becomes a perfect sphere, and the curly

Table 1 Symbols used in the mathematical descriptions of membrane stress

\begin{tabular}{|l|l|}
\hline Symbol & Reference \\
\hline$R$ & External radius \\
\hline$r$ & Internal radius \\
\hline$w$ & Membrane thickness \\
\hline$l$ & Longitudinal semi-axis \\
\hline$p$ & Transmural pressure \\
\hline$t_{\text {hoop max }}$ & Maximum hoop stress \\
\hline$t_{\text {meridional max }}$ & Maximum meridional stress \\
\hline\{\} & \{Shape determinant $]$ \\
\hline
\end{tabular}

bracket term attains its minimum value of 0.5 , a value that is characteristic of a sphere.

Applied to the ovoid (spheroid) shapes used to emulate each ampulla, membrane theory indicates that hoop stress is maximal at the equator. Its value is given by Eq. 3 :

$$
t_{\text {hoop } \max }=\left\{1-r^{2} / 2 l^{2}\right\}(r)(1 / w)(p)
$$

Note that when the $r$ and $l$ are equal, the prolate spheroid is a perfect sphere and the curly bracket term has a value of 0.5 , the minimum value characteristic of a sphere as noted above.

In contrast, when $l$ is much larger than $r$, the prolate sphere becomes very elongated and virtually cylindrical and the curly bracket term assumes a value of 1.0, the value characteristic of a cylinder. Applied to the cylinder shape used to emulate the utricle and the crus commune, thin membrane theory indicates that maximal hoop stress occurs, shown by Eq. 4:

$$
t_{\text {hoop } \max }=\{1.0\}(r)(1 / w)(p)
$$

Note that the curly bracket term is 1.0 , the value noted above as characteristic of a cylinder.

Applied to the torus shape used to emulate the semicircular canal and the cochlea duct, thin membrane theory indicates that maximal tensile stress occurs along the innermost margin due to its maximal anticlastic shape at this location. Its value is given in Eq. 5 :

$$
t_{\text {hoop } \max }=\{1 / 2(2-r / R) /(1-r / R)\}(r)(1 / w)(p)
$$

Note that when the value for external radius $R$ is very high, the torus' major circumference becomes so large that any section effectively becomes cylindrical and the curly bracket term approaches a limiting value of 1.0 , the value associated with a cylinder. In contrast, when $R$ is smaller and approaches the limiting value of $r$, the torus is more tightly wound, and the value of the curly bracket term rises without limit, and stress mounts in parallel.

\section{Results}

The resultant findings for stress proclivity in the model chambers of the mammalian labyrinth are presented in - Table 2. These results indicate an unexpected findinga surprising consistency in the configurational features that control maximal chamber stress. Stress proclivity in every chamber is controlled not by one feature but by the product of three determinants-membrane thickness $(w)$, chamber size $(r)$, and geometric shape indicated by the term in curly brackets $(\{\})$. This means that thin membranes alone are not solely responsible for the heightened stress that must logically precede distention. Chamber size and geometry also play a role and could theoretically offset (or aggravate) any vulnerability associated with membrane thickness.

The effect of membrane thickness on stress proclivity as shown in - Table $\mathbf{2}$ is an inverse rather than a proportionate one. Thus although monotonic, it is not linear. Sharp increases 
Table 2 Chamber features and the respective determinants of stress proclivity

\begin{tabular}{|l|l|l|l|l|l|l|}
\hline Chamber name & Chamber shape & Chamber proportions & Stress proclivity & \multicolumn{2}{|l|}{ Stress determinants } \\
\cline { 5 - 8 } & & & & Thickness & Size & Membrane shape \\
\hline Saccule & Discoid & $r>>>I$ & $t / p=$ & $1 / w$ & $r$ & $\{r / 2 /\}$ \\
\hline Ampulla 1 & Oblate spheroid & $r>I$ & $t / p=$ & $1 / w$ & $r$ & $\{r / 2 /\}$ \\
\hline Ampulla 2 & Perfect sphere & $r=I$ & $t / p=$ & $1 / w$ & $r$ & $\{0.5\}$ \\
\hline Ampulla 3 & Prolate spheroid & $r<I$ & $t / p=$ & $1 / w$ & $r$ & $\left\{1-r^{2} /\left.2\right|^{2}\right\}$ \\
\hline Utricle & Perfect cylinder & $r<<<I$ & $t / p=$ & $1 / w$ & $r$ & $\{1.0\}$ \\
\hline Semicanal & Toroid & $R$ constant & $t / p=$ & $1 / w$ & $r$ & $\{1 / 2(2-r / R) /(1-r / R)\}$ \\
\hline Cochlea & Spiroid & $R$ varies & $t / p=$ & $1 / w$ & $r$ & $\{1 / 2(2-r / R) /(1-r / R)\}$ \\
\hline
\end{tabular}

in stress proclivity will occur as membrane thickness declines, and conversely stress will lessen as thickness grows. It is also important to point out that the thickness measurement refers to the load-bearing portion of the membrane. Generally, this implies the thickness of the collagen coat layer in the pars superior. In the pars inferior, it refers to the type IV collagen of the epithelial basement membrane.

The effect of radial size on stress proclivity in $\mathbf{- T a b l e ~} \mathbf{2}$ is a linear one, the larger the radial size of the chamber, the greater the maximal stress proclivity. Radial size is measured at the chamber's equator. Thus for the saccule, it is disk radius; for the spheroid and cylinder, it is the cross-sectional radius at midsection; for the toroid and spiroid, it is the internal radius of the tube. Although the figure may have larger or smaller dimensions in other planes, it is radial size that determines stress proclivity.

The effect of shape on membrane stress proclivity in -Table $\mathbf{2}$ is more nuanced and depends on the character and degree of membrane curvature (clasis). ${ }^{8}$ (Recall that when both curvatures are in the same direction, as in a sphere, the membrane is designated synclastic; when there is only one curvature, as in a cylinder, it is uniclastic; and when curvatures are opposing, as along the inner margin of a torus, it is anticlastic.) The greater the net curvature in a membrane, the greater its mechanical advantage and the lower the attendant membrane stress proclivity. ${ }^{9}$ Thus the sphere can be expected to exhibit the lowest shape determi- nant of stress because its two principal curvatures share stress equally. In all other structures, the secondary curvature is less than the primary with a consequent rise in stress along the primary curvature.

The effect of shape on stress proclivity can be exemplified by assigning specific proportions to the various chamber geometries and then computing a numerical value for each chamber's shape determinant as shown in - Table $\mathbf{3}$. These numerical values can then be plotted to give a graphic depiction of the effect of chamber shape on stress proclivity as shown in - Fig. 1. This figure clearly illustrates the nadir shape value of 0.5 associated with a perfect sphere and the modest doubling to 1.0 associated with cylinders. However, beyond this central nadir, stress proclivity rises abruptly for the more extreme shapes in the spectrum. The flattened discoid shape of the saccule is associated with heightened stress proclivity at one end of the shape spectrum. At the other end, the spiral torus exhibits increasing stress proclivities as the spiral tightens and its inner margin becomes more anticlastic. The specific shape value for any individual labyrinth will depend on its numerical dimensions.

\section{Discussion}

Lord Kelvin noted that "in physical science the first essential step in the direction of learning any subject is to find principles of numerical reckoning and practicable methods

Table 3 Shape contribution to stress proclivity based on specific chamber proportions

\begin{tabular}{|l|l|l|l|}
\hline Element geometry & Shape formula & Relative proportions & Shape determinant value \\
\hline Discoid & $r / 2 l$ & $r: I=10: 1$ & 5.0 \\
\hline Oblate spheroid & $r / 2 l$ & $r: I=2: 1$ & 1.0 \\
\hline Sphere & $r / 2 l$ & $r: I=1: 1$ & 0.5 \\
\hline Prolate spheroid & $1-r^{2} / 2 I^{2}$ & $r: I=5: 6$ & 0.7 \\
\hline Cylindroid & $1-r^{2} / 2 l^{2}$ & $r: I=1: 100$ & 1.0 \\
\hline Basic toroid & $1 / 2(2-r / R) /(1-r / R)$ & $r: R=1: 2$ & 1.5 \\
\hline Tight toroid & $1 / 2(2-r / R) /(1-r / R)$ & $r: R=8: 9$ & 5.0 \\
\hline
\end{tabular}




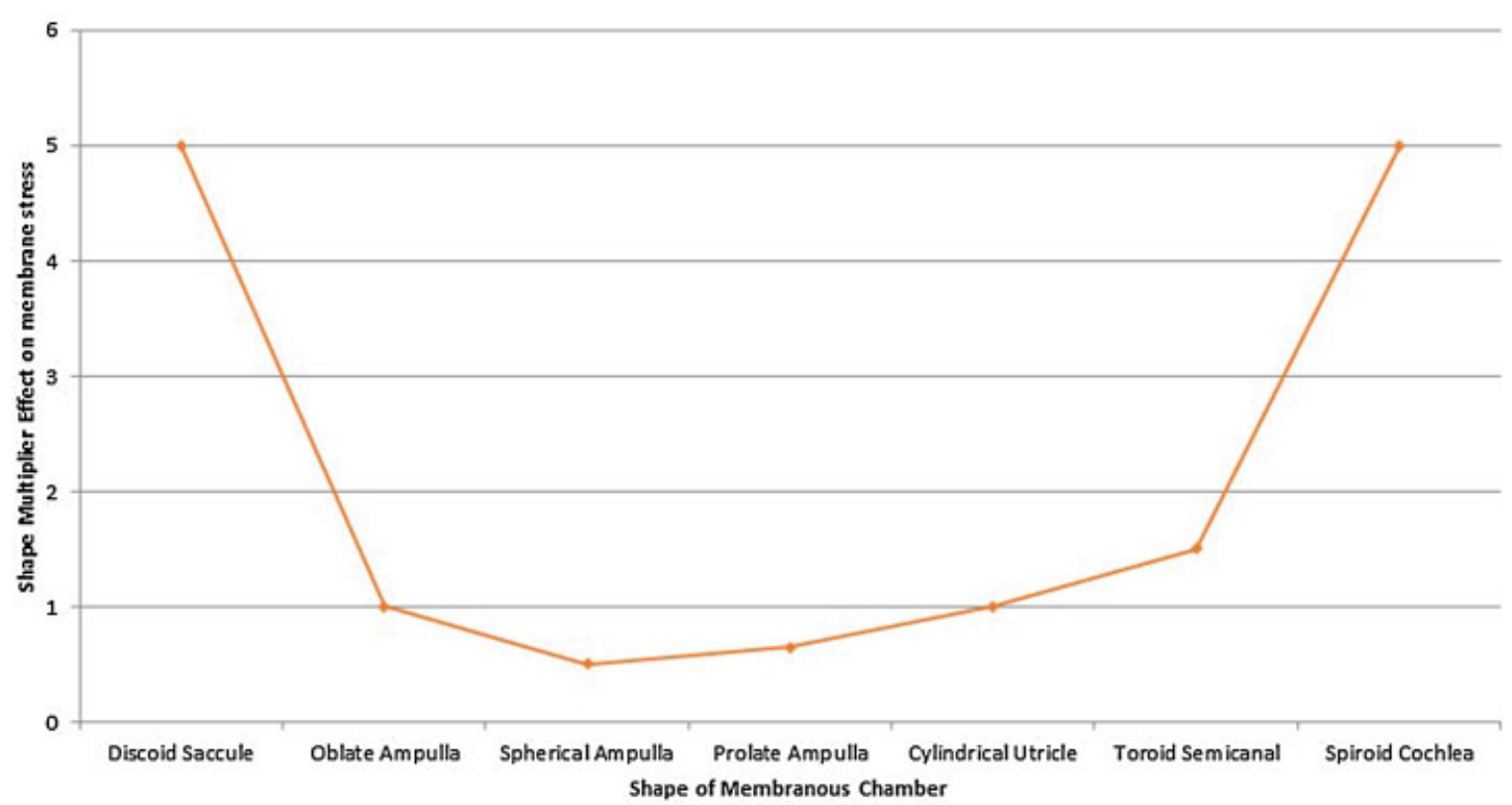

Fig. 1 The graph shows how the shape of a particular chamber affects the stress proclivity of the membrane, based on the relative proportions presented in -Table 3.

for measuring some quality connected with it."10 Application of the principles of thin membrane theory to the chambers of the mammalian labyrinth permits the inherent limits of intuition to be addressed by providing quantitative estimates of the stresses born by microscopic structures under load.

Stress proclivity as defined previously refers to the tendency to develop membrane stress in the face of raised endolymphatic pressure. However, it does not imply that elevated stress actually exists in the labyrinthine membranes. In mathematical terms, stress proclivity is the first order differential of stress with respect to pressure. This feature derives from the constitutive static relationship that applies to the membranes at all times and at all locations throughout the labyrinth. ${ }^{6}$ It is an inherent property of the physical structure of the labyrinth and is present even if no transmural pressure exists. An alternate way to think about it is to presume that the very slightest pressure is present, one so slight as to be virtually unmeasureable and with an associated membrane distention that is imperceptible. In this case, the stress proclivity determination will show how incipient stress will be distributed, what chambers will experience high stress and which low.

Previous work in this area used a simple model of the superior labyrinth that indicated significant disparities in stress proclivity in the toadfish labyrinth. ${ }^{8,11}$ To represent the vestibular chambers in the toadfish, that model used spheres and cylinders, singular ideal geometries in the overall shape landscape with invariant and relatively low shape determinant values ( 0.5 and 1.0 , respectively). Based on such configurations, that model provided a conservative lower bound for the shape contribution to membrane stress proclivities but could not determine if shape departures from those ideals were associated with substantial elevations in stress above the nadir values implicit in the sphere and cylinder model elements.

This study probes those configurational parameters that control stress in the more elaborate structures of the mammalian labyrinth. All the structures of the evolutively older pars superior exhibit configurations (spheres, cylinders, and thin tori) that have shape determinants that lie near the nadir. They also appear to have thicker membranes and small chamber sizes. In fact, the semicircular canal has the smallest internal diameter and therefore smallest radial size of all the vestibular chambers. Such a permutation of determinants implies that the membranes of the pars superior enjoy a mechanical advantage when it comes to resisting the distensive influence of transmural pressure.

This feature of the pars superior is probably a reflection of the greater evolutionary age of its structures, which first appeared in primitive fish during the Ordovician period more than 400 million years ago. ${ }^{12}$ Their attendant low membrane stress proclivities imply membrane stability and may thus help explain the stable configuration that the pars superior has attained over time and across species. One investigator opined that when it came to the structure of the superior labyrinth, They were made right for all time. ${ }^{13}$

In contrast, the structures of the newer pars inferior have developed more recently with the appearance of terrestrial life and the eventual evolution of mammals. The geometric figures that characterize the saccule and the cochlea duct of the mammalian inferior labyrinth (discoid and spiroid) are seen to be those with the higher shape determinant values. Furthermore, these chambers exhibit thin membranes that are stress prone. ${ }^{14}$ And these chamber membranes are relatively flat, implying larger radial size values that increase stress proclivity. Thus for all three determinants of stress proclivity, the membranes of the inferior division exhibit a 
permutation of determinant values that place them at a mechanical disadvantage in responding to transmural pressure. Their projected greater stress proclivities and attendant higher risk of deformation may indicate a lesser degree of stability and thus potential for evolutionary change.

\section{Conclusions}

Membrane thickness is not the sole determinant of stress proclivities in the membranes of the mammalian labyrinth. Rather, such proclivity is the interaction of thickness, size, and shape. This interaction could theoretically achieve low and uniform stress levels throughout the labyrinth though compensating determinant values chamber by chamber. How nature actually manages this permutation of determinants in any particular species can be determined based on actual measurements of that species' labyrinth membranes. The presence of hydropic distention in the human, the guinea pig, and the cat suggest that these species will have high stress proclivity values for the pars inferior chambers.

In summary, these considerations suggest that the thick, highly curved synclastic membranes of the smaller chambers that are characteristic of the superior labyrinth in mammals are less prone to stress. Conversely, the thin, flat, and anticlastic membranes that are characteristic of the inferior labyrinth are apt to develop high stress levels in response to endolymphatic pressure. This heightened proclivity to stress may play a role in the pars inferior's greater potential for hydropic distention.

\section{References}

1 Pender DJ. A geometric appraisal of the labyrinthine membranes in mammals. Otorinolaringologia. 2013;63:85-97

2 Schuknecht HF, Gulya AJ. Endolymphatic hydrops. An overview and classification. Ann Otol Rhinol Laryngol Suppl 1983;106:1-20

3 Kimura RS. Experimental blockage of the endolymphatic duct and sac and its effect on the inner ear of the guinea pig. A study on endolymphatic hydrops. Ann Otol Rhinol Laryngol 1967;76(3): 664-687

4 Hallpike CS, Cairns H. Observations on the pathology of Ménière's syndrome: (Section of Otology). Proc R Soc Med 1938;31(11): 1317-1336

5 Pender DJ. A Model Design for the Labyrinthine Membranes in Mammals. Laryngoscope 2014;124:E245-E249

6 Fung YC. Biomechanics: Mechanical Properties of Living Tissues. New York, NY: Springer-Verlag; 1993

7 Fryer DM, Harvey JF. High Pressure Vessels. New York, NY: Chapman \& Hall; 1998

8 Pender DJ. A model analysis of static stress in the vestibular membranes. Theor Biol Med Model 2009;6:19

9 Nave CR. Hyperphysics. Available at: http://hyperphysics.phy-astr. gsu.edu/hbase/ptens3.html. Accessed on July 26, 2014

10 Pender DJ. Electrical Units of Measurement, Popular Lectures and Addresses 1889;1:80-81

11 Pender DJ. A model analysis of tensile stress in the toadfish vestibular membranes. Int J Otolaryngol 2011;2011:519293

12 Kuraku S, Kuratani S. Time scale for cyclostome evolution inferred with a phylogenetic diagnosis of hagfish and lamprey cDNA sequences. Zoolog Sci 2006;23(12):1053-1064

13 Guggenheim LK. Phylogenesis of the Ear. Murray \& Gee: Culver City, 1948

14 Okuno T, Sando I. Localization, frequency, and severity of endolymphatic hydrops and the pathology of the labyrinthine membrane in Menière's disease. Ann Otol Rhinol Laryngol 1987;96(4):438-445 